\title{
Conversion of aspergilloma to chronic necrotizing pulmonary aspergillosis following treatment with sunitinib: A case report
}

\author{
YEON WOOK KIM ${ }^{1}$, HYUN WOO LEE ${ }^{1}$, JAEYOUNG CHO ${ }^{1}$, HAN-SOL CHOI $^{1}$, JUNGSIL LEE $^{1}$, \\ SUNG SOO PARK ${ }^{1,2}$, EUN YOUNG HEO ${ }^{1,2}$, KWANG NAM JIN ${ }^{3}$ and DEOG KYEOM KIM ${ }^{1,2}$ \\ ${ }^{1}$ Division of Pulmonary and Critical Care Medicine, \\ Department of Internal Medicine, Seoul National University College of Medicine; \\ ${ }^{2}$ Division of Pulmonary and Critical Care Medicine, Department of Internal Medicine, ${ }^{3}$ Department of Radiology, \\ Seoul Metropolitan Government Seoul National University Boramae Medical Center, Seoul, Republic of Korea
}

Received December 15, 2014; Accepted December 30, 2015

DOI: $10.3892 / \mathrm{ol} .2016 .5052$

\begin{abstract}
Semi-invasive or invasive aspergillosis occurring following chemotherapy with sunitinib is a rare condition with unknown incidence and prognosis. Here, we report a case involving a 59-year-old male who had a history of underlying stable aspergilloma and was newly diagnosed with metastatic renal cell carcinoma. Following surgical resection for renal cell carcinoma and adjuvant chemotherapy with sunitinib for 8 months, the patient presented with hemoptysis. Chest computed tomography revealed an increased soft tissue mass and air crescent sign of the underlying aspergilloma, combined with consolidation and bronchial artery hypertrophy around the lesion. The patient underwent bronchoscopy with a biopsy of the lesion and was eventually diagnosed with chronic necrotizing pulmonary aspergillosis, which had progressed from the underlying stable aspergilloma. This case highlights the fact that clinicians should be aware of the risk of opportunistic conversion from stable aspergilloma to invasive fungal infections in patients undergoing sunitinib treatment.
\end{abstract}

\section{Introduction}

Semi-invasive aspergillosis, also known as chronic necrotizing pulmonary aspergillosis, is a form of aspergillosis infection in which the fungus demonstrates an intermediate phenotype between a simple saprophyte and an invasive pathogen (1). This form of aspergillosis infection is extremely rare in patients with normal immunity. The major risk factor triggering invasiveness of aspergillosis is the significant compromise of

Correspondence to: Professor Deog Kyeom Kim, Division of Pulmonary and Critical Care Medicine, Department of Internal Medicine, Seoul Metropolitan Government Seoul National University Boramae Medical Center, 20 Boramaero-5-Gil, Dongjak-Gu, Seoul 156-707, Republic of Korea

E-mail:kimdkmd@snu.ac.kr

Key words: pulmonary aspergiollosis, sunitinib, opportunistic infection immune function due to various insults, including underlying immunodeficiency or malignancy, cytotoxic chemotherapies and conditioning regimens for solid organ or hematopoietic stem cell transplantation. These insults are also known to contribute to poor patient outcome $(2,3)$.

Sunitinib, an indolin-2 analog that inhibits various types of receptor tyrosine kinases (RTKs), is currently the treatment of choice in patients with metastatic renal cell carcinoma and imatinib-resistant gastrointestinal stromal tumors $(4,5)$. Adverse effects including cytopenia, diarrhea, nausea, skin symptoms and abnormal heart functions are reported, but opportunistic fungal infection caused by sunitinib treatment is a rare condition of unknown incidence and prognosis $(5,6)$. Here, we report a case of pulmonary aspergilloma that progressed to semi-invasive aspergillosis following sunitinib treatment. Written informed consent was obtained from the patient for this study.

\section{Case report}

A 59-year-old male had previously been diagnosed with stable aspergilloma in the upper lobe of the left lung and was undergoing routine follow-up and observation of the size of the fungal ball (Fig. 1A). After 7 months, the patient presented with chest discomfort and was eventually diagnosed with renal cell carcinoma of the right kidney, with distant metastasis to the left eighth rib. He underwent right radical nephrectomy with chest wall resection. After recovering from surgery, he started adjuvant chemotherapy with sunitinib. This treatment was continued for 8 months.

During the ninth month of sunitinib therapy, hemoptysis developed and reached a level of $\sim 400 \mathrm{ml}$ in the last 10 days of treatment. Physical examination revealed decreased breathing sounds in the left upper lung. Blood tests revealed decreased hemoglobin levels $(9.9 \mathrm{~g} / \mathrm{dl})$ and increased C-reactive protein levels $(3.91 \mathrm{mg} / \mathrm{dl})$. Prothrombin time, activated partial thromboplastin time, fibrinogen levels and liver function tests were all within the normal ranges. Chest computed tomography revealed interval development of paramediastinal consolidation and bronchial wall thickening in the left upper lobe, with an increased soft tissue mass and air crescent sign in the underlying aspergilloma lesion. Left bronchial artery hypertrophy 
A

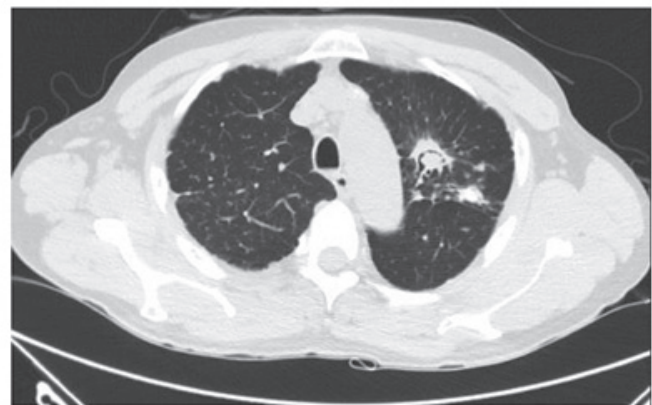

B

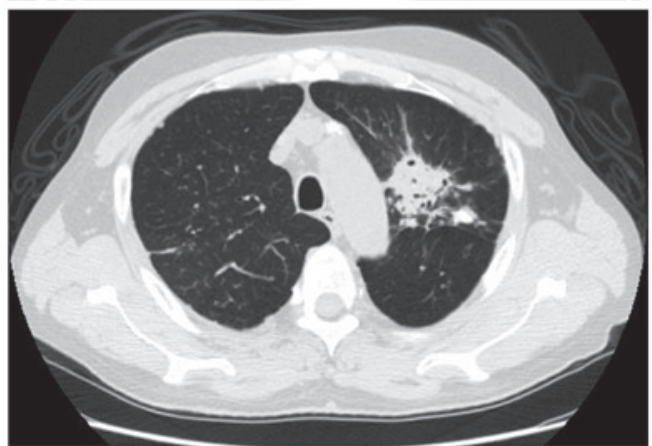

C

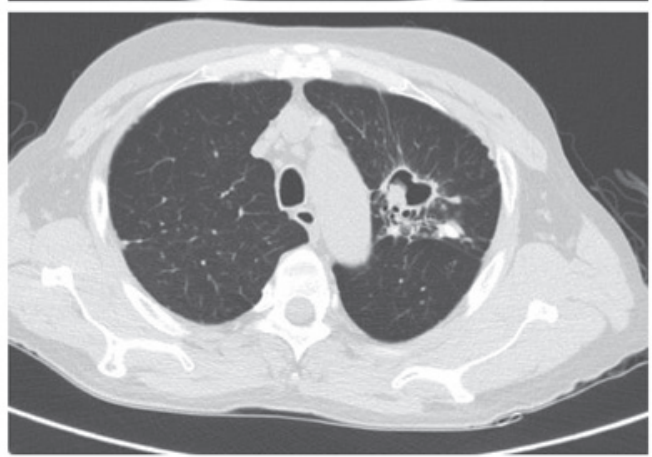

Figure 1. (A) Chest computed tomography demonstrating an irregular cavitary mass and air crescent sign in the left upper lobe, consistent with stable aspergilloma. (B) Chest computed tomography demonstrating bubbly opacity surrounding the underlying aspergilloma with paramediastinal consolidation and bronchial wall thickening in the left upper lobe. (C) Chest computed tomography following treatment with voriconazole and clinical improvement, demonstrating decreased consolidation and a reduction in inflammatory nodules around the aspergilloma.

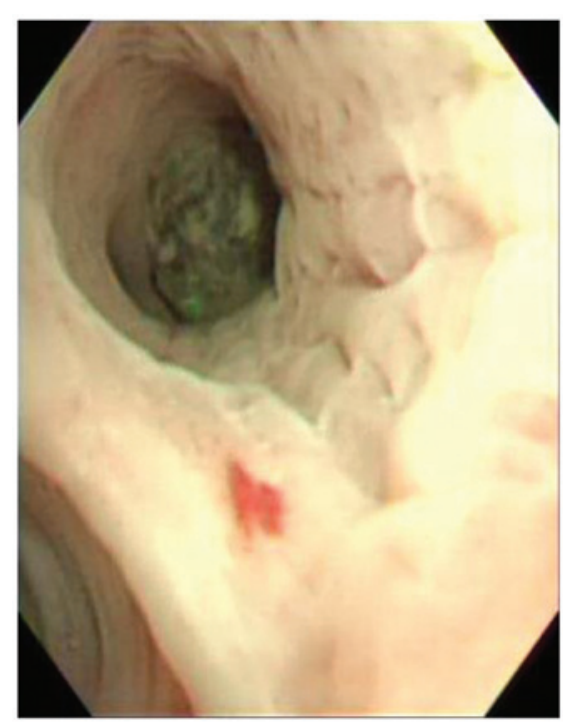

Figure 2. Bronchoscopy demonstrating a round, black fungal ball in the upper division of the apicoposterior segment of the left upper lobe.
A

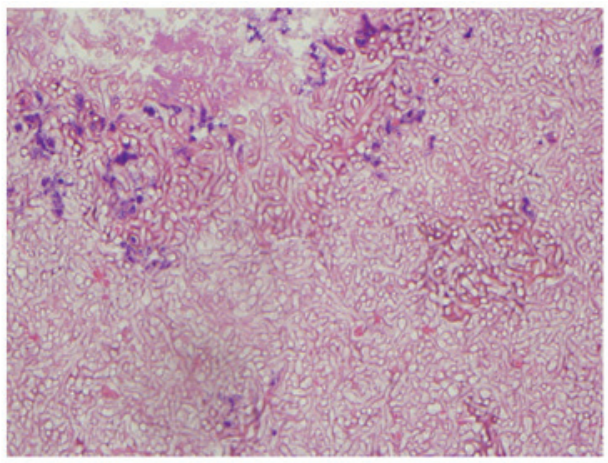

B

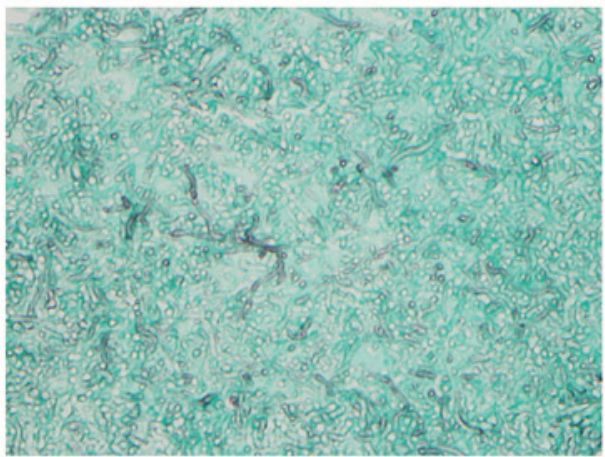

C

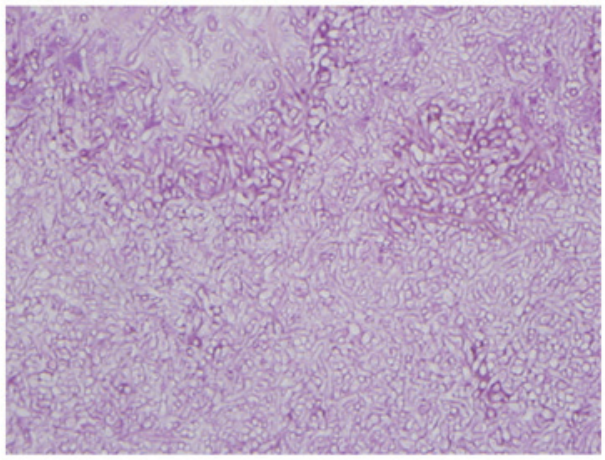

Figure 3. Microscopic findings in the biopsied tissue obtained during bronchoscopy, revealing numerous fungal hyphae morphologically consistent with aspergillosis. (A) Hematoxylin and eosin staining; magnification, x400. (B) Gomori methenamine silver staining; magnification, x400. (C) Periodic acid-Schiff staining; magnification, x400.

was also observed (Fig. 1B). Bronchial artery embolization was performed to control hemoptysis. Upon bronchoscopic examination, a round, black fungal ball was noted to be impacted in the apicoposterior segment of the upper division of the left upper lobe (Fig. 2). An endoscopic biopsy was taken from the visible mass. Pathological examination including Gomori methenamine silver and periodic acid-Schiff staining revealed numerous fungal hyphae, which were morphologically consistent with aspergillosis (Fig. 3).

Sunitinib therapy was discontinued, and the patient was started on treatment with oral voriconazole (200 mg bid). Treatment with voriconazole led to an improvement in his symptoms and radiological resolution of consolidation around the aspergilloma. A cavity remained in the left lung following antifungal treatment (Fig. 1C).

\section{Discussion}

The typical niches of Aspergillus are air, soil, food and decaying organic material. The conidia of Aspergillus are 
easily dispersed into the air and when they reach a permissive environment, such as the lung of an immunosuppressed patient, they germinate into hyphae, the invasive form of Aspergillus (7). The virulence of aspergillosis depends on the immune status of the host, but leads to poor outcome in patients with significantly compromised immune function, regardless of the cause (3). Compared with the notably high incidence and mortality of invasive aspergillosis among patients undergoing cytotoxic regimens during hematopoietic cell transplantation, invasive and semi-invasive forms of aspergillosis are relatively rare in patients undergoing targeted chemotherapy with RTK inhibitors. To date, there has been only one reported case of invasive aspergillosis occurring following treatment with sunitinib (5).

In the current case, the patient had underlying stable pulmonary aspergilloma prior to the diagnosis of renal cell carcinoma and sunitinib treatment. In the majority of patients, stable aspergilloma does not change in size or invade the surrounding tissue. It is reported to spontaneously resolve or decrease in size in $\sim 13 \%$ of cases (8). Therefore, stable aspergilloma does not usually require active treatment. During follow-up, an increase in size or conversion into a semi-invasive/invasive form of aspergillosis is rare $(8,9)$.

In the present case, stable aspergilloma progressed and converted to semi-invasive aspergillosis after sunitinib therapy was adopted. For the treatment of semi-invasive aspergillosis, antifungal agents including voriconazole, amphotericin B and itraconazole should be used promptly. Surgical resection should be considered for patients with recurrent hemoptysis and those intolerant or resistant to antifungal therapy (9).

Sunitinib inhibits various RTKs including vascular endothelial growth factor receptors 1 and 2, platelet-derived growth factor receptors and fibroblast growth factor receptor 1, leading to a $40-44 \%$ partial response rate in metastatic renal cell carcinoma patients (10). Previous studies also suggest that sunitinib inhibits the phosphorylation of signal transducer and activator of transcription 3 (Stat3) and Src-tyrosine kinase, suppressing the response of Th-1-type cytokines and SYK-tyrosine kinase, which uses Stat3 as a substrate. Th-1-type cytokine responses and SYK-dependent signals are known to be significant in the mechanism of host defense against Aspergillus and other fungi (5). Therefore, it is likely that inhibition of Stat3 by sunitinib plays a key role in the development of aspergillosis infections in patients taking this medication.

The current case suggests that clinicians should be aware of the potential risk of opportunistic fungal infections in patients undergoing sunitinib treatment. In particular, patients with underlying stable aspergilloma who are planning to undergo sunitinib therapy should be warned of the risk of conversion into semi-invasive or invasive pulmonary aspergillosis. In such patients, monitoring of the symptoms of invasive aspergillosis and radiological changes in the underlying stable aspergilloma is required.

\section{References}

1. Gefter WB, Weingrad TR, Epstein DM, Ochs RH and Miller WT: 'Semi-invasive' pulmonary aspergillosis: a new look at the spectrum of aspergillus infections of the lung. Radiology 140: 313-321, 1981.

2. Salgado SM, Costa CA, Buqalho AA, Semedo JA, Ribeiro JC and Carreiro LM: Semi-invasive aspergillosis in an immunocompetent patient with Swyer-James-MacLeod syndrome: a case report. J Med Case Rep 4: 153, 2010.

3. Upton A, Kirby KA, Carpenter P, Boeckh M and Marr KA: Invasive aspergillosis following hematopoietic cell transplantation: outcomes and prognostic factors associated with mortality. Clin Infect Dis 44: 531-540, 2007.

4. Chow LQ and Eckhardt SG: Sunitinib: from rational design to clinical efficacy. J Clin Oncol 25: 884-896, 2007.

5. Visvardis EE, Gao F, Paes MN, Duprez O and Waxman J: Lung Aspergillosis in renal cell carcinoma patient treated with sunitinib. QJM 105: 689-692, 2012.

6. Hoshino Y, Hasegawa H, Ishii Y, Endo T, Ochiai H, Okabayashi K, Kaneko G, Mikami S, Mukai M, Oya M and Kitagawa Y: Two cases of bowel perforation associated with sunitinib treatment for renal cell carcinoma. Int J Clin Oncol 17: 412-416, 2012.

7. Garcia-Vidal C, Viasus D and Carratalà J: Pathogenesis of invasive fungal infections. Curr Opin Infect Dis 26: 270-276, 2013.

8. Lee JK, Lee YJ, Park SS, Park JS, Cho YJ, Park YS, Yoon HI, Lee CT and Lee JH: Clinical course and prognostic factors of pulmonary aspergilloma. Respirology 19: 1066-1072, 2014.

9. Cabral FC, Marchiori E, Zanetti G, Takayassu TC and Mano CM: Semi-invasive pulmonary aspergillosis in immunosuppressed patient: a case report. Cases J 2: 40, 2009.

10. Goodman VL, Rock EP, Dagher R, Ramchandani RP, Abraham S, Gobburu JV, Booth BP, Verbois SL, Morse DE, Liang CY, et al: Approval summary: sunitinib for the treatment of imatinib refractory or intolerant gastrointestinal stromal tumors and advanced renal cell carcinoma. Clin Cancer Res 13: 1367-1373, 2007. 